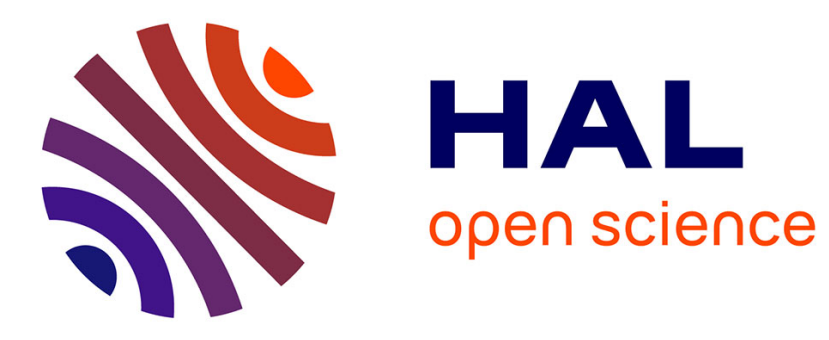

\title{
L'anthropologie dans l'espace européen de la recherche
} Irène Bellier

\section{To cite this version:}

Irène Bellier. L'anthropologie dans l'espace européen de la recherche. Séminaire "Actualités de l'anthropologie", co-organisé par l'Association des anthropologues (AFA) et l'association pour la recherche en anthropologie sociale (APRAS), séance "L'anthropologie dans l'UE" coordonnée par Irène Bellier, MSH, Jun 2007, Paris, France. halshs-00157432

\section{HAL Id: halshs-00157432 \\ https://shs.hal.science/halshs-00157432}

Submitted on 26 Jun 2007

HAL is a multi-disciplinary open access archive for the deposit and dissemination of scientific research documents, whether they are published or not. The documents may come from teaching and research institutions in France or abroad, or from public or private research centers.
L'archive ouverte pluridisciplinaire HAL, est destinée au dépôt et à la diffusion de documents scientifiques de niveau recherche, publiés ou non, émanant des établissements d'enseignement et de recherche français ou étrangers, des laboratoires publics ou privés. 
l'anthropologie dans la politique européenne de recherche

Séance coordonnée par Irène Bellier avec la participation comme intervenants de :

Isabelle Bruno ; Benoît de l'Estoile ; Aziliz Gouez; Marie-Dominique Ribereau Gayon ; Jan

Spurk

\section{Intervention de Irène Bellier}

Indépendamment des questions qu'elle peut poser aux anthropologues en tant que citoyens, inscrits dans un espace politique et conscients des enjeux de la mondialisation comme de la libéralisation, l'Europe pénètre l'anthropologie en France de deux manières :

1) par la voie des réformes macro impulsées dans le cadre de la stratégie de Lisbonne et $\mathrm{du}$ processus de Bologne. Ce cadre européen des réformes de la recherche et de l'enseignement ont un impact sur les réformes nationales des mêmes secteurs que l'on a abordées lors des précédentes séances du séminaire. Ces réformes s'inscrivent dans la volonté affichée de mettre sur pied l'économie de la société de la connaissance la plus dynamique au monde (sic).

2) par la voie des programmes-cadres (dit PCRD - on est au $7^{\text {̀̀me }}$ PCRD), lesquels répondent à une architecture tout à fait particulière pour laquelle deux efforts doivent être accomplis pour que les anthropologues trouvent leur place : en amont dans la définition des thématiques ; en aval dans le montage des projets.

Les deux voies ont un effet sur les réformes nationales concernant l'infrastructure des institutions de recherches, l'emploi des chercheurs et enseignants ainsi que sur l'allocation des moyens de recherche. Sur ces bases, on peut formuler des observations ou des critiques sur le caractère finalisé, inadéquat ou ne tenant pas compte des perspectives «uniques» de l'anthropologie. Il faut aussi se demander ce que l'Europe peut apporter, comment on peut utiliser les opportunités qui s'ouvrent à travers la facilitation des échanges entre centres et labo, c'est à dire se demander aussi comment devenir pro-actifs et être capables de proposer, par exemple des thématiques ou des modalités de recherche qui soient plus proches de ce que l'on sait faire ou que l'on veut améliorer.

1) En termes de perspectives de recherche susceptibles d'être portées au niveau européen pour permettre aux anthropologues de tous les Etats membres de se retrouver dans les programmes qui sont définis.

2) En termes de modalités de fonctionnement qui seraient mieux adaptées à nos manières de travailler : pour les anthropologues la question lancinante reste celle du « terrain », son financement, son intégration dans les parcours, sa valorisation, son éthique, etc.

Cela suppose de se penser comme acteur collectif - ce qui n'est pas gagné dans notre domaine, pour faire pression sur le gouvernement dont les projets de réforme du secteur de la recherche ne sont guère sensibles aux particularités des SHS. Cela passe à mon avis par le développement de relations plus soutenues avec les autres disciplines des SHS. Ce même genre de raisonnement se déploie ailleurs en Europe, comme le montre le contenu d'une conférence à Oxford, fin juin ; why isn't anthropology widely accepted by policy makers ? ${ }^{\mathrm{i}}$ 
Cela nous conduira à explorer l'Espace européen de la recherche qui se met en place, ses objectifs, ses cadres structurants - avec le Conseil européen de la recherche et les programmes cadres.

La réalisation de l'EER passe par la mise en place des « centres d'excellence », c'est-à-dire le regroupement en réseaux d'institutions de recherche de pointe. Ce qui oblige à se demander où se localise l'anthropologie dans cette reconfiguration, compte tenu des réformes en cours.

- Faut-il un centre d'excellence en anthropologie en France, pour le mettre en rapport avec d'autres de même type ailleurs en Europe ? et qui ? Cela doit se penser en rapport avec d'une part les thématiques de recherche, le caractère proprement scientifique de nos apports, d'autre part en rapport avec la question de la mobilité avec la mise en place de la notion de chercheur européen, lequel répond à une perspective idéaltypique -imaginée par le cadre européen - en rupture avec le modèle national de chercheur que l'on connaît. Le caractère positif ou dommageable de la réforme doit être justement appréhendé.

- Faut-il négocier une place pour l'anthropologie dans des centres d'excellence pluridisciplinaire, à quel prix et comment? C'est tout l'enjeu de la place de l'anthropologie dans les réformes résultant du processus de Bologne, mais c'est aussi une question d'agenda et de perspectives scientifiques de recherche qui est ici posée. Sachant la rareté du nombre de postes offerts au recrutement et la complexité de la fabrication de tels centres d'excellence, quelles sont les perspectives scientifiques de recherche et d'enseignement qui figureront dans de tels centres d'excellence ? La structuration de la discipline en France, les modes de recrutement ainsi que les rapports de force entre spécialistes disons des aires culturelles et nouveaux objets de l'anthropologie sont au cœur du problème, dans une dynamique qu'il faut concevoir comme " ouverte" sur les problèmes de société et sur les changements que l'on observe dans le monde.

C'est dans cet espace qu'il me semble important de réfléchir, non pas à courte vue de la prochaine commission du CNRS ou session de recrutement des universités mais à moyen et long terme ; 1) pour ne pas casser des dynamiques de recherche qui nous distinguent au plan de la qualité au niveau international ; 2) pour ne pas casser les dynamiques de recherche qui renouvellent les apports de l'anthropologie.

On voit combien il est important de s'intéresser à ces aspects de politique européenne : ils configurent le cadre dans lequel se produisent toutes les réformes que l'on doit apprécier pour positionner le futur de l'anthropologie en France, comme en Europe.

Ce sont des politiques, des stratégies, des processus largement initiés par la Commission européenne qui définissent un cadre macro relativement coercitif dans lequel nous devrions nous constituer comme acteurs, en sachant que ce n'est pas la profession elle-même qui est appelée à poser ses observations, demandes ou critiques directement au niveau européen. Celles-ci transitent par différents comités d'experts et par les positions défendues par les représentants du gouvernement dans les instances européennes.

\section{Il y a donc plusieurs enjeux :}

- comprendre le cadre global dans lequel les métiers de la recherche et de l'enseignement sont appelés à se repenser en incorporant dans nos perspectives toute la dimension technologique qui amène à réformer nos pratiques (structuration de 
réseaux virtuels, utilisation des bases de données on line, mise en archives ouvertes des résultats, etc, système de référencement mais aussi accessibilité des données, et évaluation... : en ayant présent à l'esprit la contrainte linguistique.

- Voir les dimensions concernant le métier de chercheur, la carrière, l'emploi, les rapports avec les bailleurs de fonds, les rapports secteur public et privé dont le détail est fourni dans la Charte européenne de recrutement.

- évaluer dans les propositions qui sont faites, ce qui affecte les SHS et l'anthropologie en particulier. Il faut voir dans le cadre structurant proposé ce qui peut être éventuellement utile - on évoque par exemple le rôle des régions. Mais aussi ce qui peut être perturbant, par ex. la question de l'autonomie et de la mise en concurrence des pôles d'enseignement et de recherche qui risquent de pousser à une régionalisation de nos travaux voire à handicaper la poursuite des recherches sur le reste du monde, en étant inscrit dans une communauté scientifique large - je vois ici des contradictions fortes dans la perspective européenne. Comment dans le contexte d'une territorialisation des politiques de recherche penser la circulation des thématiques et des enseignants chercheurs? Ce qu'il faut penser pour l'anthropologie c'est une circulation des chercheurs, une ouverture des terrains d'étude dans le cadre d'une réciprocité

- former un groupe de travail pour se donner une voix et constituer un interlocuteur aux fonctionnaires de la Commission, dans le cadre de ce que l'on peut comprendre comme du lobbying, lequel sert à défendre ses intérêts dans un univers de décision complexe, partagé par la représentation sectorielle (le monde de la recherche) et la représentation nationale (l'Etat). La commission a lancé un livre vert en janvier 2007 sur l'évaluation de l'EER: Quelle réponse des associations, des disciplines, quelle consultation des chercheurs?

Cela représente pour nous, dans le cadre de ces assises, 2 perspectives-clés

- rassembler les anthropologues dans une seule association professionnelle qui se doterait des moyens de penser les enjeux professionnels en tenant compte des spécificités de la recherche et de l'enseignement en anthropologie

- raccrocher plus fermement cette association à l'association européenne des anthropologues (EASA) et aux diverses structures qui se sont mis en place pour représenter les chercheurs, les étudiants, les enseignants, au niveau européen.

La séance est construite autour des interventions de :

Isabelle Bruno, maître de conférence à Lille 2, en sciences politiques; sur la Stratégie de Lisbonne,

Jan Spurk, enseignant à Paris V, en sociologie : sur l'idéologie de la politique européenne

Marie Dominique Ribereau Gayon, anthropologue à l'université d'Oslo ; sur "La demande de recherche en anthropologie dans les APR européens : qui y répond en France? ".

Aziliz Gouez, anthropologue à la Fondation Notre Europe ; sur la place des anthropologues dans un tel organisme et la demande européenne en sciences sociales et en anthropologie. 

12 juin 2007

Benoit de l'Estoile, anthropologue, prof à l'ENS, membre du comité exécutif de l'EASA sur les relations entre les anthropologues au niveau européen (réseaux et médiation).

Si le temps le permet, je dirais un mot sur la Charte européenne du recrutement, les questions de code éthique, et je reviendrais sur l'expérience des programmes européens de Recherche.

Robert Gibb de l'université de Glasgow, interviendra dans la discussion ainsi que tous les membres de l'assemblée ici réunie.

\footnotetext{
${ }^{\mathrm{i}}$ As an academic discipline, Anthropology is equipped for a thorough understanding of the increasing frictions and problems in today's shrinking world. Its ethnographic approach and historical awareness provide for a thick nterpretation of the interweaving of causalities that spark clashes or power abuses. And yet this perception and meticulousness does not inform the broader 'ecology of ideas' that influences both public opinion and governmental policy makers. While many anthropologists are involved in agencies and foundations with handson projects or policies, our discipline has not made the major contributions its intellectual power calls for. It is still easy for powerbrokers to ignore reports that few people are aware of. This conference is a platform for a dialogue about ways in which anthropological research on current global conflicts can engage a world of asymmetric power relations. Mediating understandings may offer an opportunity for a rich dialogue that could reduce tensions and apprehensions. It is not simply about writing popular books, but rather about engaging in a comprehensible dialogue with other disciplines and perspectives.

Keynote speaker: Prof. Thomas Hylland Eriksen (University of Oslo)

Distinguished speaker :: Prof. Akhil Gupta (UCLA)

Guest speakers :: Prof. Keith Hart (Goldsmiths College, University of London) :: Prof. Mohammad Talib (University of Oxford) :: Prof. Sam Beck (Cornell University) :: Dr. Stef Jansen (University of Manchester) :: Dr. David Gellner (University of Oxford) :: Dr. Gerhard Anders (University of Zurich) :: Dr. Laura Rival (University of Oxford)
} 